\title{
‘Habitat’ Suitability Index Mapping for Industrial Symbiosis Planning
}

\author{
Paul D. Jensen, Lauren Basson, Emma E. Hellawell and Matthew Leach
}

\section{Summary}

By 'working with the willing', the National Industrial Symbiosis Programme (NISP) has successfully facilitated industrial symbiosis throughout the UK and, in the process, delivered significant economic and environmental benefits for both Programme members and the country as a whole. One of the keys to NISP's success is that, unlike failed attempts to plan and construct eco-industrial systems from scratch, the Programme works largely with existing companies who have already settled in, developed, and successfully operate within a given locale. This article argues that existing and mature industrial systems provide the best prospects for identifying opportunities for, and ultimately facilitating, industrial symbiosis.

Due to levels of diversification and operational fundamental niches that, in the fullness of time, develop within all industrial systems, industrially mature areas are deemed to be industrial symbiosis 'conducive environments'. Building on the conservation biology concept of a habitat suitability index, the article presents a methodology for comparing a potential site for eco-industrial development, to a known baseline industrial 'habitat' already identified as being highly conducive to industrial symbiosis. The suitability index methodology is further developed and applied to a multi-criteria-evaluation geographic information system to produce a 'habitat' suitability map that allows practitioners to quickly identify potential industrial symbiosis hotspots (the methodology is illustrated for England). The article concludes by providing options for the development of symbiosis suitability indices and how they can be used to support the facilitation of industrial symbiosis and regional resource efficiency. 


\section{Introduction}

Industrial symbiosis can be seen as the establishment of close working agreements between normally unrelated industrial (or other) organizations that lead to resource efficiency (Jensen et al. 2011a). Working agreements can, for example, involve the innovative reuse of one company's by-products as another's raw material; the sharing of power, water and/or steam supplies; and/or the simple sharing of manufacturing capacity, logistics, and/or expertise.

Reasons for the self-organized evolution or planned implementation of industrial symbiosis working agreements, otherwise known as synergies, are manifold. Apart from the business imperative of needing to improve profitability and competitiveness, drivers of symbiosis can also be social, environmental, or regulatory in nature (Chertow 2007). Regardless of the initial driver behind synergy genesis, the implementation of industrial symbiosis has, importantly, been shown to generate significant environmental and economic benefits both for the companies involved in a given synergy, and the wider communities in which they reside (see Laybourn and Morrissey 2009).

The most prominent and widely cited example of industrial symbiosis derives from the instances of self-organized symbioses observed within the industrial district of Kalundborg, Denmark. The extensively studied network of synergies that exist within Kalundborg, which largely developed in reaction to economic forces and local scarcity of resources (Ehrenfeld and Gertler 1997), have served as both a study site for researchers looking to understand the mechanisms behind the organic evolution of industrial ecosystems, and as the inspiration behind planned attempts to create so-called eco-industrial parks.

Though studying the industrial symbiosis networks within Kalundborg (and other similarly self-organized sites) has improved the academic understanding of industrial symbiosis development, attempts to implement these lessons in the form of premeditated eco- 
industrial parks have largely failed (Gibbs and Deutz 2005, 2007; Chertow 2007). Reasons offered for their failure are manifold; however, the difficulties involved in generating intercompany cooperation, which is required for companies to commit to the linking of production processes (Gibbs 2003), is widely held to be one of the primary barriers to their development (see Gibbs and Deutz, 2007, for a more complete discussion on the relative success and failure of planned eco-industrial parks).

Instead of attempting to plan and construct eco-industrial parks from scratch, the United Kingdom (UK) has employed an alternative model of proactive industrial symbiosis implementation. Treating the whole country as a 'virtual' eco-industrial park, the National Industrial Symbiosis Programme (NISP) works with existing companies (and inward investment enquires) to identify business development opportunities that are capable of delivering resource efficiency. Via one-to-one engagement or multi-attendee workshops, NISP facilitates industrial symbiosis on what can best be described as a company have-want 'dating-agency' basis. 'Working with the willing', NISP enrolled over a five year period (April 2005 - March 2010) approximately 13,000 companies to the Programme. This duly facilitated a national network of synergies that has generated, among other outputs, more than $£ 1.5$ billion in collective cost savings and new sales, diverted 35 million tonnes ${ }^{1}$ of materials from landfill, and reduced carbon emissions and virgin material use by 30 million and 48 million tonnes, respectively (Laybourn and Morrissey 2009).

The key commonality that arguably characterizes the way in which NISP successfully operates and the way in which the likes of the organic symbioses at Kalundborg successfully developed is the fact that both networks are largely built around existing companies who have cemented their respective places in their markets and successfully adapted to their local environments. By working with existing companies, industrial symbiosis practitioners, or any inadvertent symbiosis facilitators, know exactly what they are working with and consequently 
what can or cannot be feasibly achieved. Unlike in the case of planned 'greenfield' ecoindustrial parks, that have tended to fail, there is little or no guesswork and synergies are not developed on platforms of aspiration or eco-industrial desirability. Similar to biological symbiosis, opportunities for industrial symbiosis that exist between established companies derive from tangible environmental (in the widest sense) pressures. There is no desirability within 'nature'; symbiotic activity within all systems is, ultimately, a response to the wider environmental influences of a maturing system.

It is thus sensible for NISP, and similar organizations, to continue to primarily work with existing industry in the pursuit of developing industrial symbiosis best practice. One option open for Programme development is the wider promotion of multi-partner utility based industrial networks. However, unlike failed attempts to plan the likes of a new Kalundborg from scratch, NISP affiliated researchers have actively sought to identify existing industrial areas that are prime for reorganization toward greater resource efficiency. By identifying and working in mature areas that are currently facing localized operational pressures, of one form or another, NISP and local stakeholders would aim to ease these pressures via the facilitation of industrial symbiosis. After alleviating the operational pressures faced by a given cluster of industries, it would be expected that individual companies within a given locale would be open to engaging in further opportunities for company specific synergies. NISP experience has shown that once a company is involved in the successful implementation of a synergy, it becomes more open and willing to engage in dialogue relating to all manner of symbiotic working opportunities.

This article continues by presenting an industrial symbiosis decision-support methodology inspired by the conservation biology concept of a Habitat Suitability Index (HSI). It takes the idea of a HSI, transfers it to a mature industrial system, and presents a Geographic Information System (GIS) mapping methodology for the identification of 
industrial 'habitats', within England, that are outwardly prime for eco-industrial development. It is suggested that the presented methodology can be used by NISP for the effective deployment of practitioners and by similar organizations wishing to initiate or develop industrial symbiosis in other countries. Furthermore, there is the potential for the mapping process to be used to assist regional planning, as it can identify which type of development different regions are suited to (i.e., building on the idea of a 'conducive environment'). This promotes context sensitive development, which is considered to be more likely to succeed than development based on visions that may be misaligned with the resources available within a given area.

\section{Habitat Suitability Mapping}

Brief Overview of the Habitat Suitability Index Methodology

The concept of a habitat suitability map derives from the conservation biology sciences and is an advancement on the long-held practice of employing a HSI to conduct an assessment of a given area's capacity to perform a particular role. Whether employed for simple habitat inventory purposes or targeted species translocation, HSIs have been used since at least the $1970 \mathrm{~s}$ as a means of assessing the relative suitability of a habitat to sustain and promote the proliferation of a given species (see USFWS 1981). By performing an assessment of a baseline area already deemed suited to the existence of a particular organism, characterization of what are deemed to be key site variables allows a holistic picture to be painted of what makes an area conducive to the presence of the given species under investigation. For instance, key site variables could be deemed to be the quality and type of favored foodstuffs of a given organism, the quality and availability of water, and/or the prominent vegetation type and structure. Once baseline variables are selected and characterized, they are assigned relative suitability scores on a continuous scale from 0 
(unsuitable) to 1.0 (optimal) and aggregated to create an index. The level of complexity (e.g., suitability scales, potential weighting, extent of compensation) incorporated into the assignment of an individual variable suitability score, or aggregated HSI, is only limited by the level of variable detail a given researcher's resources will allow him or her to acquire. Comparing the suitability index acquired for the baseline with another area on a ratio basis (i.e., study site conditions $\div$ baseline optimum conditions), an assessment can be made as to how similar, and thus how suitable for a given purpose, the new area is to the HSI baseline. Where study site conditions exactly match those of the measured baseline optimum, the HSI ratio score equals 1 . As HSIs are inevitably bespoke in nature, and hence developed on a context-specific basis, the calculated HSI ratio for an area can be allocated to various discrete categories of relative suitability; for example, from zero $(0)$ to low $(<0.5)$ to high $(\geq 0.5)$ (e.g. Wakeley 1988) $)^{2}$.

With advancements in computing capability and the development of sophisticated GIS software, it has become increasingly popular for habitat suitability assessments to be made via the production of a habitat suitability map. By producing a GIS model consisting of map layers pertaining to the relevant variables of a given HSI, the HSI assessment can be largely automated and a map can be produced that provides a visual depiction of the results of the assessment. In effect, the maps depict all sites within a predetermined geographic area that possess the same niche characteristics as the study baseline. The production of suitability maps allows targeted and strategic habitat management to be conducted over wider geographic scales than is generally the case with manually conducted site wide HSI assessments. 


\section{Industrial Symbiosis and Suitability Mapping}

By characterizing the historically evolved natural and manmade characteristics of an industrial area, that is known to possess many instances of existing and potential industrial symbiosis, it is argued that HSI methodologies can be used to identify similarly industrialized areas that may also be likewise predisposed to eco-industrial development. An industrial symbiosis HSI, or Symbiosis Suitability Index (SSI), is conducted as per equation (1) and, as within a traditional HSI assessment, can provide a direct comparison between any industrialized area and the key characteristics of a baseline of 'optimal' industrial symbiosis potential.

(1)

$$
\mathrm{SSI}=\frac{\sum_{i=1}^{n} w_{i} V_{S C i}}{\sum_{i=1}^{n} w_{i} V_{O C i}}
$$

Where $n$ is the number of suitability index variables, $w_{i}$ is (if employed) the weighting factor assigned to a given variable, $V_{S C i}$ is the individual scores for study area variable characteristics, and $V_{O C i}$ is the individual scores for variable characteristics considered to be optimum. Where the sums of study area characteristics and baseline optimum characteristics are identical, SSI optimality is observed (i.e., SSI = 1).

Similar to how traditional HSI assessments have been applied to the production of habitat suitability maps within the conservation sciences, it is argued that the SSI process can be largely automated by applying its assessment of baseline variables to a GIS to produce a symbiosis 'habitat' suitability map. Although there are few published articles on the subject, 
research into the use of GIS as a decision-support tool for industrial symbiosis planning and facilitation is not new. Nobel and Allen (2000), Kincaid and Overcash (2001) and Özyurt and Realff (2002) provide details on the use of GIS to model options for water recycling and reuse in Pasadena, Texas, USA; to aid the identification and facilitation of by-product exchanges within North Carolina, USA; and for the development of an agricultural-industrial ecosystem within Georgia, USA, respectively. Additionally, Massard and colleagues have made presentations at several international conferences on the subject of GIS and the role it can play within proactive industrial symbiosis facilitation (e.g., Massard and Erkman 2007; Massard et al. 2009).

Multi criteria 'habitat' suitability mapping techniques that incorporate the aggregation of multiple raster maps depicting key variables and their suitability values have, however, not been used as a decision-support tool within the context of industrial symbiosis facilitation. In comparison to 'dots on a map', an holistic insight into the relative suitability of an area to perform a given objective can be discerned from multi criteria overlay maps, thus making a suitability map a valuable and much used tool within conservation research circles. Indeed, habitat suitability maps, like HSIs, have been widely employed as a decision-support tool within applied ecology research for many years (see Kliskey et al. [1999] for extensive reference to species-specific mapping models).

Similar to the production of a traditional HSI, the methodology and criteria used to produce GIS-based suitability maps is highly context-specific and highly conducive to bespoke development. A common drawback to the development and production of suitability maps is, however, the existence and availability of sufficiently detailed data covering wider geographic areas. If datasets were available that detailed, for example, the location and excess utilities capacity of all companies on a national scale, the process of pointing practitioners directly to areas with the same characteristics as a utilities-based SSI baseline 
could be fully automated via the symbiosis suitability mapping element of the SSI assessment. The likelihood of being able to generate or acquire such datasets is, however, minimal. Thus, not wishing to restrict the geographic scale that the suitability map (presented later) covers, the Symbiosis Suitability Map (SSM) acts as a screening tool that narrows down sites within a given study area (in our case, England) that are outwardly similar to the chosen suitability baseline. Once relative suitability hotspots have been identified by the mapping process, a more detailed in-the-field SSI comparison to the baseline of optimality, which goes beyond an assessment of the historically evolved geospatial characteristics of the 'habitat' being compared, would need to be conducted to confirm suitability. The methods employed to produce a SSM are detailed below.

\section{Demonstration of Symbiosis Suitability Mapping}

\section{Suitability Baseline: South Humber Bank}

To produce an SSI capable of identifying industrial areas within England that are conducive to eco-industrial development in the form of utilities sharing, the baseline chosen to determine optimality was the South Humber Bank (SHB) industrial region. The SHB industrial region, which currently covers approximately 29 square kilometres $\left(\mathrm{km}^{2}\right)^{3}$, lies adjacent to the Humber estuary and straddles the two counties of North and North East Lincolnshire within northern England (figure 1). The site is known to have attracted 'modern' industrial activity to the area since at least the early 1950s. 


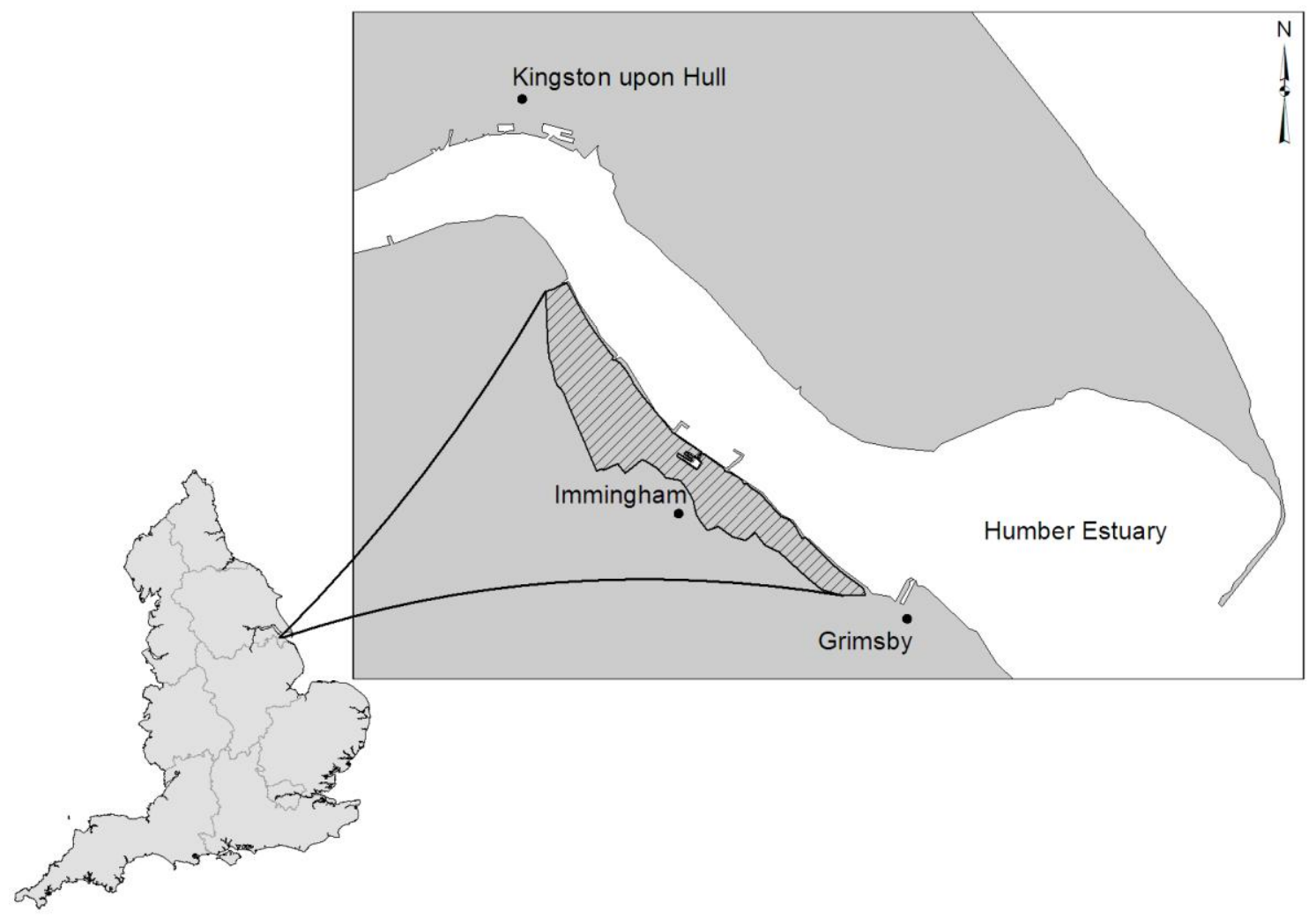

Figure 1 England and the South Humber Bank industrial district (hatched area).

The industry that populates the SHB is largely made up of two oil refineries, several chemical processing and production plants, and the numerous support services that are typically associated with an active deep-water port facility - notably, it is the UK's largest port area by tonnage. The area is further populated by food processing plants which were largely drawn to the area on the back of the remnant skills of the fishing industry and its ancillary services that, until the 'Cod Wars' of the 1970s, dominated much of the Humber region.

Over the years, numerous instances of both self-organized and facilitated industrial symbiosis have taken place on the site $^{4}$. At the time of writing, at least five companies are known to be involved in 'organically' evolved donor dependent power and steam sharing. While, to meet both existing and prospective companies' increasing and new demands for power and steam, 16 further SHB companies, NISP, and other local stakeholders have been 
involved in proposals for the wider sharing of excess utilities capacity. Materials and service based synergies taking place on the site are many and diverse among NISP's SHB based members - ranging from the simple sharing of land to the innovative reuse of biofuel production by-products.

The existing instances of industrial symbiosis, and the increasing potential for further power and steam cascading on the SHB, can be said to have followed phases similar to those witnessed within biological ecosystems that are subject to primary colonization, system succession, and diversification. Oil and chemical industries were largely attracted to the SHB due to its proximity to a deep-water estuary that historically has been used to both discharge effluents and import the necessary bulk resources from around the world required for oil refining and many forms of chemical processing. The site also consequently acts as an export point for bulk products.

The process of companies colonizing the SHB industrial area, to exploit the benefits of its geographic location, systematically led to an increase in the demand for primary operational resources (e.g., power and water $)^{5}$. As the site continued to mature, companies wishing to locate to the $\mathrm{SHB}$, and any in-situ energy intensive companies that wished to develop their operations, were (and are) subject to any restrictions that might emanate from infrastructure constraints and the fluctuating power (and water) production and demand trends of existing site occupants.

Similar to within a biological ecosystem, where the forces of succession can make a given habitat less conducive to the development of many primary colonizers, the continued colonization and maturation of the site has, on occasion, restricted individual company and site-wide development. Much like within the Kalundborg municipality, to find solutions to their respective utilities-based constraints on development both individual and collections of SHB companies and other organizations have, at times, chosen to adapt to the prevailing 
conditions by engaging in symbiotic activities (ranging from the previously mentioned power and steam sharing to the multi partner uncovering of several million tonnes of grey water to facilitate past SHB growth demands).

\section{Exploiting the 'Conducive Environment'}

The self-centric utilization of resources that has latterly restricted some SHB based companies' colonization or development aspirations is arguably the potential driver behind the communal eco-industrial development of the area. Though companies can and have solved development problems through new or upgraded utilities delivery infrastructure, from an industrial symbiosis perspective, the more logical option for developing the SHB (and similarly evolved 'habitats') would be to tap into known instances of excess utilities capacity that many companies have acquired over years of implementing operational efficiencies and/or changes in production methods. By exploiting both the negative and positive aspects of a maturing industrial system (e.g., possible restrictions to power and water usage, and evolved operating efficiencies leading to excess utilities capacity, respectively), opportunities intrinsically exist, via each company's fundamental niche ${ }^{6}$, for eco-industrial development. This scenario, in part, is the basic process of ecological succession toward system optimization via increased niche complementarity and niche facilitation. For the SSM presented here, areas of mature energy intensive industrial development, which are prime for utilities sharing, constitute an industrial symbiosis 'conducive environment'. As possibly one of the UK's most mature (energy-centered) industrial areas, the SHB is thus considered a good baseline for the SSI assessment ${ }^{7}$. 


\section{Selection of Symbiosis Suitability Index Variables}

At this point it should be reemphasized that the following assessment of variables, and the description of 'habitat' suitability mapping, was conducted with the specific intention of identifying areas similarly suited to utilities sharing as the SHB. The methodology described for producing the SSM is not aimed at identifying areas best suited to industrial symbiosis in the form of material by-product exchanges (though the final SSM does identify such areas). As with all forms of GIS work, the choice of variables employed within the SSM is dictated by the availability of suitable data. As such, the variables chosen to produce the SHB SSM are derived from data sources that are readily accessible to, in this case, industrial symbiosis practitioners based in England. The relative weight assigned to individual variables was determined by the specific goal of the SSM and is detailed within the map layer aggregation section of the article. The nine variables chosen to characterize the SHB 'habitat' are presented below.

\section{Combined Heat and Power}

As the SSI model is primarily aimed at the replication of utilities sharing schemes that currently (and potentially) exist within the SHB industrial area, Combined Heat and Power (CHP) was deemed to be the key site variable. Industrial CHP clusters are also deemed to be indicative of heavy, energy-intensive, industry (e.g., manufacturing/processing). Within the SHB boundary there are eight known industrial CHP plants. All non industrial CHP plants (e.g., hospitals) were removed from the GIS dataset employed within the SSM. 


\section{Power Plants (Non-CHP)}

Power plants were chosen as a variable for their potential to be incorporated into power-sharing schemes. Power plants were also seen as a primary indicator of geospatial 'habitats' of heavy industry. Within the SHB boundary there are three known power plants.

\section{Boreholes}

As a prerequisite resource within almost all systems, industrial or otherwise, all sources of water are seen as key SHB SSI variables. As such, the locations of active company boreholes are included as an SSM variable. Industrial borehole licensing clusters also indicate areas of dense industrial activity. Ten live borehole access points exist within the SHB boundary.

\section{Controlled Water}

Although controlled discharges of water require remediation (possibly via industrial symbiosis), it is seen as another potential source of industrial water cascading. Clusters of Integrated Pollution Prevention and Control (IPPC) controlled water data points are also indicative of areas of heavy industry. Twenty-nine IPPC controlled water records relating to nine unique companies, exist within the SHB boundary.

\section{$\underline{\text { Coast-Estuaries-Rivers }}$}

Coastline, estuaries and rivers, apart from historically being a discharge point for effluents, are seen as a raw material and final product transport option. Estuaries are also widely associated with some forms of industry that possess a high utilities demand (e.g., in the UK, oil refineries). The SHB lies within close proximity of several primary rivers and a deep-water estuary. 


\section{$\underline{\text { Industrial Waste }}$}

Industrial waste is a source of materials for potential material based synergies. Clustered industrial waste data points also indicate high levels of localized manufacturing. Within the SHB boundary there are 165 non-recovered industrial waste IPPC records relating to 22 unique companies.

\section{Industrial Diversity}

There is evidence to suggest that clusters of diverse industry types provide greater opportunities for localized materials based industrial symbiosis (Jensen et al. 2011a); consequently an assessment of SHB diversity has been incorporated into the SHB SSI survey. Industrial diversity was calculated by attributing geographic IPPC industry type data points to England's 10km Grid Square system. For each IPPC populated grid square, the Simpson's

Diversity Index was used to calculate industrial diversity (i.e., $1-\sum(n / N)^{2}$, where $n$ is the total number of specific industry types falling within a square and $N$ is the total number of all companies falling within the grid square). Following conversion of the grid squares to discrete data points, all points with a diversity index score of less than 0.5 (i.e., those not showing the minimum characteristics of the SHB) were removed from the dataset. The mean index recorded within England was 0.2; the highest recorded index was 0.8.

\section{Primary Roads}

Primary roads are seen as essential to the transport and exchange of raw materials, byproducts and end products. Typically clusters of heavy industry also tend to be located close to primary road systems. No industrial plot within the SHB lies further than $20 \mathrm{~km}$ from a point of access to a motorway. The furthest distance from a SHB plot to a major dual carriageway is $6.6 \mathrm{~km}$. 


\section{$\underline{\text { Railway (Standard Gauge) }}$}

Railways are seen as another mode of materials (and fuel) transport, and are also a key characteristic of the SHB. Along with roads, railways are also seen as 'habitat' corridors that, within ecological research, help to promote system resistance and resilience. No company within the SHB boundary is situated more than $3.5 \mathrm{~km}$ from a railway line. The proximity and relative density of rail infrastructure is used as the characteristic SSM distance, rather than the distance to railway access points, as this provides a better representation of the evolved geospatial characteristics of the baseline. Points of railway access would be ascertained, if necessary, during a detailed site assessment.

\section{Characterization of Variables}

In the case of the SHB SSI assessment, the geospatial characterization of variables was achieved by measuring the mean average Euclidean (i.e., straight line) distance between each of the data points for each individual variable (e.g., within the SHB boundary shown within figure 1, there are eight known CHP plants and the mean distance between each plant is 6,154 meters $[\mathrm{m}]^{8}$ ). In the case of the Coast-Estuaries-Rivers and Primary Roads polyline layers, the variable characteristic was determined by measuring the largest Euclidean distance that existed between an SHB plot and the nearest polyline variable being characterized. All variable characterization values are shown in table 1. 
Table 1: Summary of SSI Variable Geospatial Characterization

\begin{tabular}{|c|c|c|}
\hline SSI Variable & Dataset Source & $\begin{array}{l}\text { Characterization } \\
\text { Distance (meters) }\end{array}$ \\
\hline CHP & DECC 2008 & 6,154 \\
\hline Power Plants (Non-CHP) & DECC 2008 & 6,416 \\
\hline Boreholes & EA $2008 b$ & 6,160 \\
\hline Controlled Water & EA 2005 & 5,369 \\
\hline Industrial Waste & EA 2008a & 5,020 \\
\hline Industrial Diversity & EA 2008a & 32,600 \\
\hline Primary Roads ${ }^{a}$ & Ordnance Survey 2009 & 6,630 \\
\hline Railways (SG) & Ordnance Survey 2009 & 3,571 \\
\hline Coast-Estuaries-River $^{b}$ & Ordnance Survey 2009 & 2,684 \\
\hline
\end{tabular}

Notes: ${ }^{\text {a. }}$ The Primary Roads map layer was produced by merging motorway and dual carriageway Ordnance Survey (2009) vector data. ${ }^{b}$ Separate coastline, estuaries and primary river layers were aggregated into one layer. Although an estuary is a key feature of the South Humber Bank, employing a separate estuaries layer would diminish the suitability of potential inland symbiosis hotspots.

\section{Variable Mapping}

Following georeferencing of all datasets and importation into the GIS software (i.e., ArcGIS 9.3s ArcMap mapping platform), vector format map layers for each discrete SSI variable were plotted onto a boundary map of England (figure 2). To produce map layers in the format required for eventual Multi-Criteria-Evaluation (MCE) mapping, the vector data for each variable was converted into raster format using the ArcMap Distance - Straight Line tool $^{9}$. The tool draws continuous raster data from each of the variables data points (or lines) outward toward the limits of the mapping boundary or up to a predefined outer radius. In the case of the presented SSM, an outer raster production radius of $32,600 \mathrm{~m}$ was employed. The $32,600 \mathrm{~m}$ outer radius boundary was derived from research showing that this is the average 
(median) distance materials tend to travel within industrial symbiosis partnerships facilitated within the UK (see Jensen et al. 2011a). By using the 32,600 m distance as the outer limit of raster production, an element of potential materials exchange planning is incorporated into the final suitability map (discussed later).

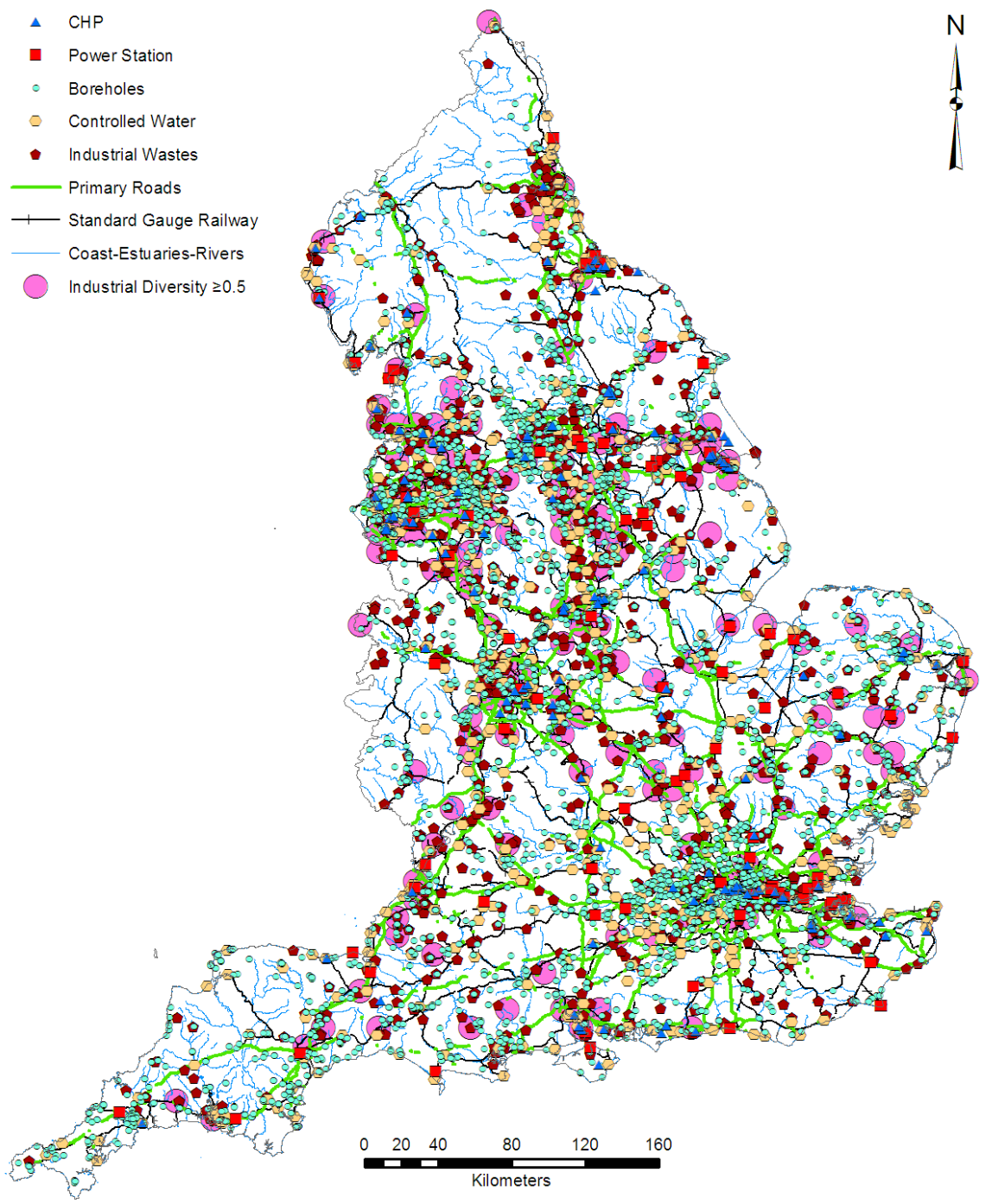

Figure 2 Map of England showing all plotted South Humber Bank Symbiosis Suitability Index variables in vector format

Note: at the scale the map is presented, it is difficult to differentiate between variables. Figure 2 is primarily presented to highlight the high number and density of chosen SSI variables in relation to the results presented within the Symbiosis Suitability Map (figure 4). 
By default, ArcMap produces ten equally spaced bands of raster cell values that radiate from the data point, or line, outward toward the mapping boundary. Each band represents a given distance from the data point or line. Where data bands from two or more data points or lines meet, the bands are simply merged and the common value of the two (or more) bands is represented rather than an aggregation or estimated interpolation of values being produced. Effectively this also provides a basic representation of variable density within a given area.

Once raster layers for all variables had been produced using the Straight Line tool, their raster cell values were reclassified based on the individual variable characterization statistics shown in table 1. For the purposes of SSI mapping, the ten default bands produced were reclassified to show only two distance categories - one being half the distance measured during the variable characterization process (e.g., in the case of CHP, 3,077 m), the other being the remaining distance up to and including the $32,600 \mathrm{~m}$ maximum layer extent (this band was given a default value of 0 ). The reason for halving the characterization distance within the reclassification process is to ensure that only data points for individual variables that are characteristic of the SHB are merged. If the full characterization distances were employed, there is the potential for bands emanating from variable data points to be merged that lay at twice the characterization distance of those found on the SHB. For example, if the full 6,154 m CHP characterization distance is employed, there is the potential for two CHP plants laying $12,308 \mathrm{~m}$ apart to be, effectively, merged; thus creating a false hotspot of suitability that is $24,616 \mathrm{~m}$ in diameter rather than the measured $6,154 \mathrm{~m}$ geospatial characterization distance. Dependant on the importance of the individual variable layer to the ultimate objective of the SSM, the variable characterization bands (i.e., the bands produced using the halved characterization distances) were duly allocated a weighted value of 1,10 , or 100 (discussed next) (see figure 3). 
As the presence of CHP is deemed to be a prerequisite variable for the development of an industrial ecosystem similar to the SHB, its characterization band was given a unique value of 1 . To aid suitability index score conversion to discrete categories of optimum, high, medium, and unsuitable, remaining variables were given scores that would allow them to be differentiated as important to a utilities-sharing project or not essential to a utilities-sharing project. Variables pertaining to water sources and non-CHP power plants were thus given a characterization score of 100 , while roads, railways, materials (i.e., industrial waste), and diversity layers were each given a characterization score of 10 .
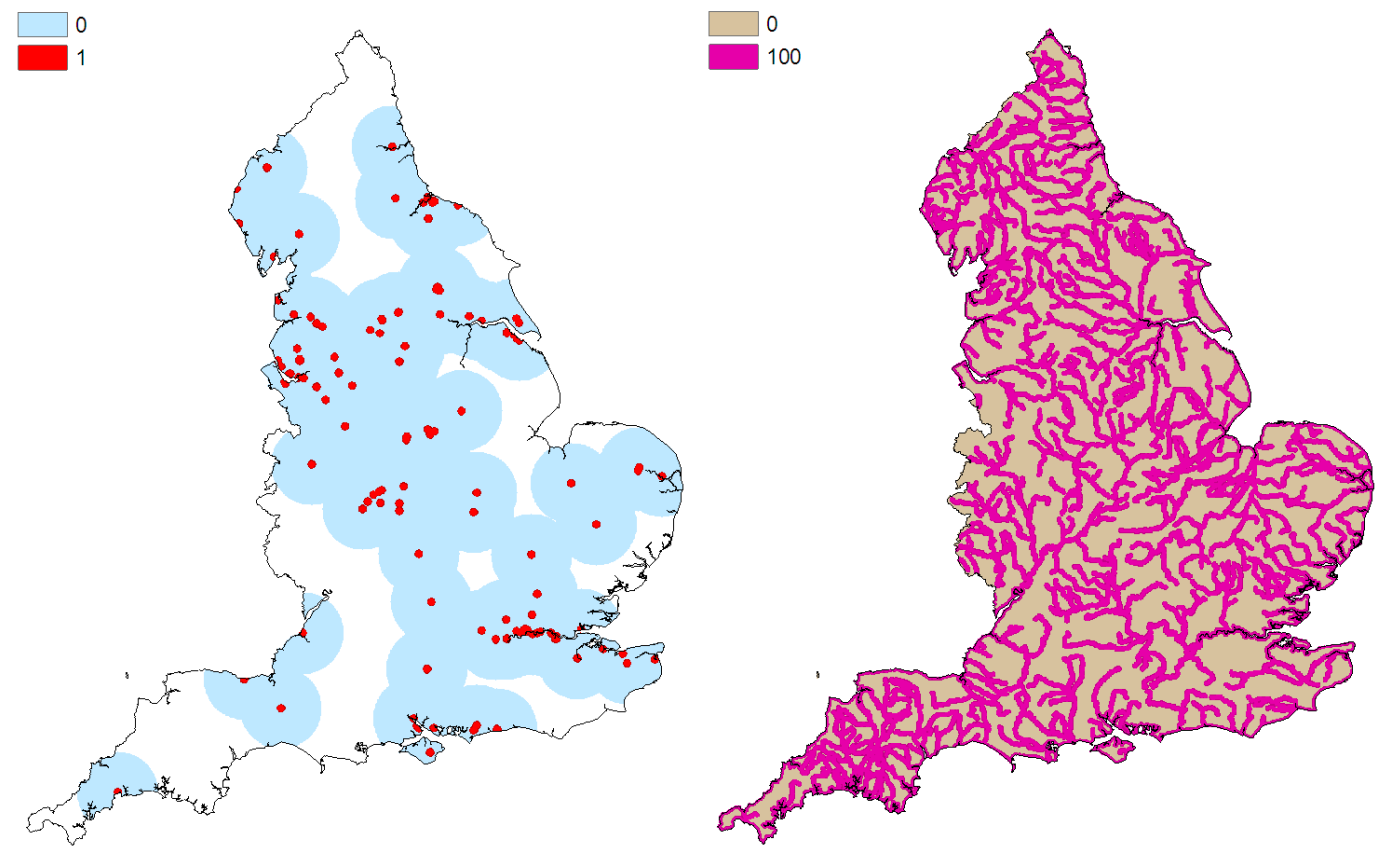

Figure 3 Examples of continuous variable data showing reclassified raster values.

Note: Left image: CHP map layer $(1=0$ to 3,077 meters and $0=3,077$ to 32,600 meters). Right image: Coast-Estuaries-Rivers map layer (100 $=0$ to 1,342 meters and $0=1,342$ to 32,600 meters).

\section{Symbiosis Suitability Index Variable Aggregation}

In developing the final SSM it was deemed that no direct weighting of map layers was required within the layer aggregation equation. Instead, relative weightings were applied 
during the map layer reclassification process (as discussed in the previous section). Allocating the unique value of 1 to CHP plants (and 0 to the outer bands of the CHP data points) allowed it to be used as a multiplier within the final SSI aggregation equation. Employing the CHP layer as a multiplier ensures that any areas that do not possess a CHP plant will be automatically depicted as being unsuitable by the output SSM (i.e., as CHP was assigned a score of 1 [optimum] and 0 [unsuitable] as its suitability scores, any area that does not possess a CHP plant will automatically be assigned a score of 0 regardless of the aggregated score achieved from other variable layers). Employing the Raster Calculator function within the ArcMap software, the reclassified SSI variable layers were aggregated in accordance with equation (2). Performing the raster aggregation calculation produces a map showing the relative suitability of a given geographic area and the raw SSI score (ranging from 0 to 440) for each industrial 'habitat' identified prior to reclassification into discrete categories, ranging from unsuitable 'habitat' to optimum 'habitat'.

$$
\mathrm{SSM}=V_{C H P} \sum_{i=1, i \neq C H P}^{i=n-1} V_{i}
$$

Where $V_{C H P}$ is the CHP SSI variable and $V_{i}$ is all remaining variables employed to produce the final SSM.

\section{South Humber Bank Symbiosis Suitability Map and Sensitivity Analysis}

The output SSM was produced by reclassifying the raw aggregation of variable map layers into four discrete categories of optimum, high, medium, and unsuitable. Areas reclassified as optimum were clearly those returning a score of 440 (or SSI = 1) (i.e., the highest score achievable after aggregation of each SHB variable). As at least one source of water was judged important for the development of utilities-sharing focused eco-industrial 
development, the areas allocated to the high suitability category were deemed to be those achieving a score equaling or between 430 and $240(\mathrm{SSI}=0.98$ to 0.55$)$ (i.e., as water sources and Non-CHP were given scores of 100, and non-essential variables were given scores of 10 , any score of 240 or greater must contain CHP, at least one source of water, and each of the variables deemed here to be associated with materials based synergies). Areas of medium suitability were deemed to be those that achieved a score equal to or between 230 and 100 $(\mathrm{SSI}=0.52$ to 0.23 ) (i.e., CHP would be present, but there is no guarantee of water or that any of the variables associated with materials based symbiosis would be present). All areas achieving a score of 40 or less (SSI $=0.09$ to 0 ) were allocated to the unsuitable category, as these areas have no known potential for water recycling and do not possess an industrial power plant. As within traditional HSI models, the allocation of scores to non optimum discrete categories can be conducted by employing numerous criteria depending on the specific purpose of the suitability modeling process; as such, the given rationale behind the SHB SSM allocation of unsuitable, medium, and high suitability categories, presented above, is not absolute and should not be interpreted as being so. It would be up to any party applying the methodology elsewhere to define criteria for the allocation of SSI scores to discrete suitability categories depending on the characteristics of the chosen baseline and the specific reason for undertaking the production of a SSM.

Although the allocation of non optimum SSM categories is important, because it provides a basic insight into the deficiencies of a particular area in relation to the specified objective of the given SSI assessment (i.e., it shows which key variables are missing from a given area), the primary purpose of the SSM is to identify areas classified as possessing the same niche characteristics as the chosen baseline 'habitat'. As such, these are the areas that, in the first instance, should be concentrated on and used to identify locations for conducting a detailed in-the-field SSI investigation. Prior to analyzing the output SSM for hotspots, 
however, a sensitivity analysis should be conducted to determine the specific effects each variable (apart from CHP) has on the depiction of the four chosen SSM suitability categories (see table 2).

Table 2: Sensitivity analysis of the effects of removing SSI variables from the SSM

\begin{tabular}{lcccc} 
& Optimum & High & Medium & Unsuitable \\
\hline Total SSM Category Identified $\left(\mathrm{km}^{2}\right)$ & 116 & 955 & 1,287 & 70,366 \\
\hline Power Plants (Non-CHP) & $189.6 \%$ & $26.2 \%$ & $-23.4 \%$ & $19.9 \%$ \\
Controlled Water & $31.5 \%$ & $5.1 \%$ & $-11.3 \%$ & $0.1 \%$ \\
Coast-Estuaries-River & $28.5 \%$ & $-21.8 \%$ & $-0.9 \%$ & $0.3 \%$ \\
Boreholes & $22.5 \%$ & $-26.3 \%$ & $-30.0 \%$ & $0.9 \%$ \\
Primary Roads & $22.0 \%$ & $0.4 \%$ & $-2.3 \%$ & $0.0 \%$ \\
SG Railways & $13.8 \%$ & $10.7 \%$ & $-9.2 \%$ & $0.0 \%$ \\
Industrial Diversity & $4.1 \%$ & $5.9 \%$ & $-0.7 \%$ & $4.5 \%$ \\
Industrial Waste & $0.4 \%$ & $-9.9 \%$ & $-7.4 \%$ & $0.0 \%$ \\
\hline
\end{tabular}

The percentage change shown in table 2 is that observed in comparison to the total area covered by each suitability category when aggregating all nine SSI variables. Apart from industrial waste and diversity, it can be seen that the removal of each variable from the layer aggregation process returned significant (i.e., >5\%) changes to the optimum suitability category within the output SSM. The simultaneous removal of both industrial waste and diversity variables led to a $6.9 \%, 17.1 \%,-9.3 \%$, and $4.5 \%$ change in the areas depicted as optimum, high, medium, and unsuitable, respectively.

Based on the results of the sensitivity analysis, it can be argued that it would be acceptable to remove the industrial waste and, possibly, diversity variables from the map layer aggregation process. If a given variable does not make any significant contribution to the final map, it could be argued that its inclusion becomes superfluous to the overall aim of the modeling exercise. The SSM, however, is designed to provide a direct geospatial 
representation of the characteristics of the chosen baseline 'habitat'. Thus all baseline features that relate to the original SSI objective should arguably be characterized and mapped. Therefore figure 4, which is derived from all nine variables, is presented as the SHB-derived SSM.

SSM: England

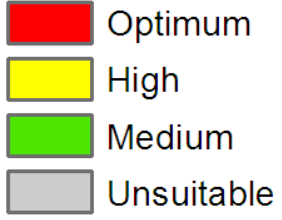

1. South Humber Bank

2. Thames Estuary

3. Port of Bristol

4. East Birmingham

5. Mersey Estuary

6. Tees Estuary
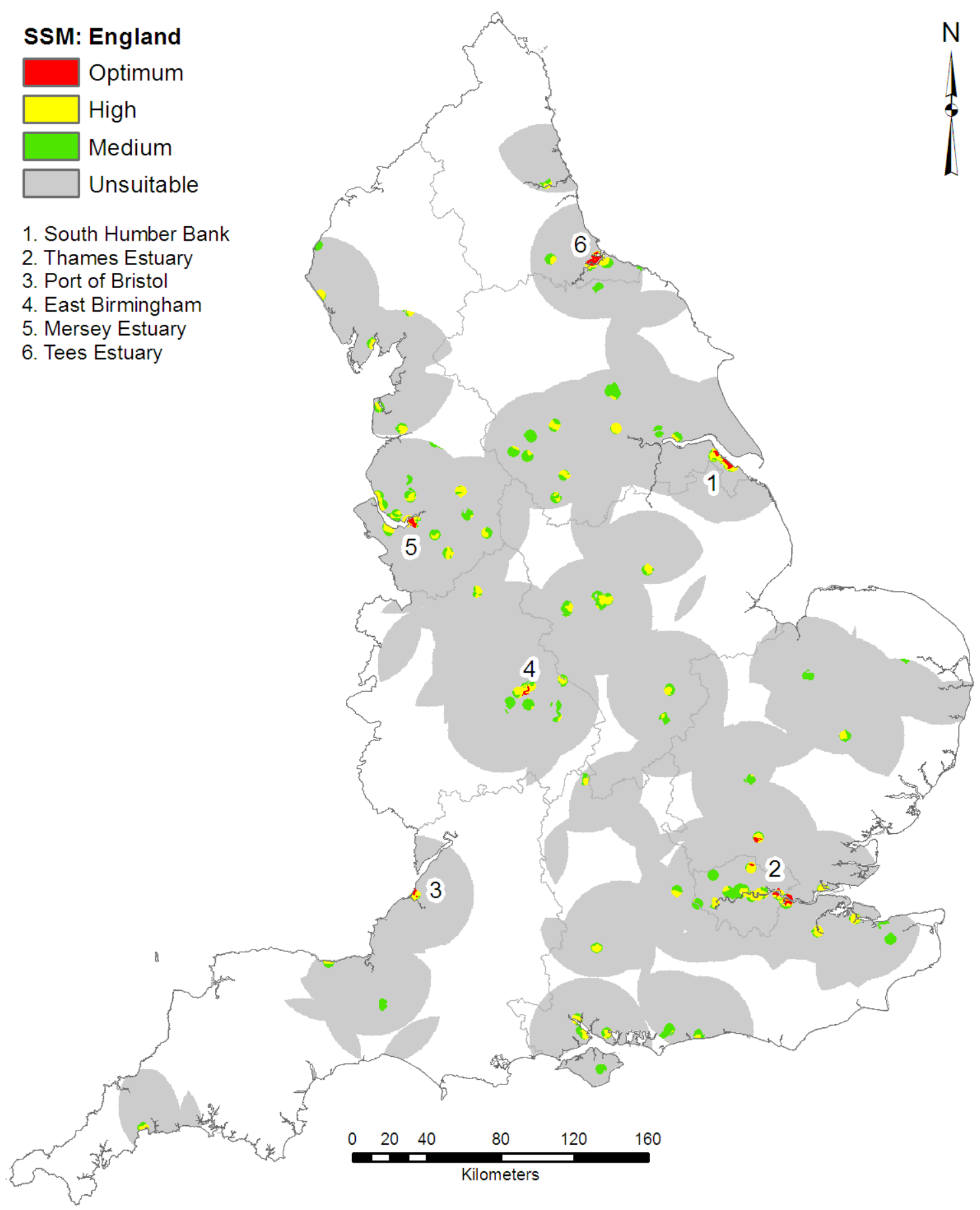

Figure 4 Symbiosis 'habitat' suitability map for England. 


\section{South Humber Bank Symbiosis Suitability Map Interpretation}

The last exercise to perform prior to deploying the SSM as a decision-support tool is to assess whether the baseline to which the rest of the map is derived is suitably highlighted. Accordingly, the SHB can be seen to be clearly displayed within figure 4 as an area for the potential development of a utilities-sharing network. Also, the fact that the suitability map has displayed the SHB as two optimum hotspots within a wider high suitability area highlights the map's accuracy, as the site does possess two dense clusters of industrial development that lie to either side of the centrally located Port of Immingham. This also highlights the need for careful consideration to be given to the choice of characterization distances. If the full variable characterization distances detailed within table 1 had been employed, the whole of the SHB and the wider surrounding area, up to the previously discussed 24,646 m, would have been wrongly categorized as being of optimum industrial symbiosis suitability. Importantly, this potential error would have been replicated for each hotspot of optimum suitability identified by the SMM, thus reducing the resolution of the SSM and its ability to direct practitioners to definitive areas of potential utilities sharing within England ${ }^{10}$.

Based on an initial assessment, the SSI evaluation methodology presents promising results. Based on the personal knowledge of the authors and discussion with local stakeholders and NISP regional teams, the Thames estuary, Port of Bristol, Mersey estuary, and Teesside are all believed to be industrially similar to the SHB, thus it is reassuring that the model has categorized these areas as being of optimum utilities-sharing suitability. The east Birmingham hotspot is arguably to be expected, as this area is historically associated with heavy industry and it is also known to be a location of numerous existing instances of industrial symbiosis. Based on an intuitive knowledge of UK industrial geography, it is perhaps surprising that other industrialized estuaries similar to the majority of hotspots 
identified by the SSM, such as the Solent on the south coast and Tyneside in north-east England, are only deemed to be of high SSI suitability, rather than the possibly expected categorization as optimum suitability. With more detailed site-based analysis, however, it was found (based on the datasets employed to produce the SSM) that the Solent and Tyneside were missing a suitable road system into the heart of the industrial area and, apart from one small CHP plant, localized power production, respectively. These findings highlight the effect of the scoring system employed to produce non optimum categories. Although a lack of power clearly precludes Tyneside from being deemed an industrial symbiosis 'conducive environment' (in terms of potential utilities sharing), questions could be justifiably raised as to whether the apparent lack of a primary road into and around the heart of the Solent's 'habitat' is sufficient to downgrade its propensity for wide-scale utilities sharing. This point illustrates the need to use the 'habitat' identification methodology as a two step process - an initial SSM screening stage and then a more detailed SSI investigation of optimal and, where appropriate, high suitability hotspots to choose locations for active practitioner engagement.

The unsuitable areas shown within the SSM essentially indicate areas that are not suited to industrial utilities sharing. However, as these areas represent the known average distance materials move from industrial clusters within the UK (e.g., Jensen et al. 2011a), these categories can be seen as the outer boundary to which a potential symbiont partner is most likely to be found for any company within an SSM hotspot that is looking to exchange material by-products. Indeed, once companies within SSM hotspots have been engaged - and in a perfect world utility sharing is facilitated - the previously stated knowledge that companies tend to be open to further industrial symbiosis activity, once they have successfully completed one working agreement, comes into play. This effectively makes the utilities-based SSM presented here a dual-purpose industrial symbiosis planning map. 
An obvious conclusion to be drawn from the SSM is that estuaries and port hinterland naturally lend themselves to the development of eco-industrial systems, as they cover the majority $(82.8 \%)$ of the combined $116 \mathrm{~km}^{2}$ of land deemed, within England, to be of optimum utilities-sharing potential. This conclusion is consistent with the known development of industrial symbiosis networks within, among other examples, Rotterdam (Netherlands), Tampico (Gulf of Mexico), Kwinana (Australia), and Kalundborg (Denmark). Notably these areas, in addition to all those identified by the suitability map, are industrially mature areas. This observation adds weight to the opening premise of this article that existing areas of mature industrial development are those best suited to eco-industrial development, due principally to the number of systematically evolved operational niches that follow the maturation and consequent development of any system (Jensen et al. 2011b).

\section{Further Development of Symbiosis Suitability Maps and Conclusions}

\section{Potential Development and Application of Symbiosis Suitability Maps}

A multitude of options exist for the development of SSMs. In parallel to the presented utilities focused SSM, the authors have developed an SSI that seeks to identify geographic areas best suited to both material exchanges in general, and material specific exchanges. Although it is in its early stages of development, the suitability hotspots produced by the materials focused SSM, which employs industrial diversity as its key variable, show high correlation to maps of existing NISP material based synergy clusters that are known to have developed over the last five years (consequently, further efforts are being placed on the development of this particular SSM).

Utilizing the SSM modeling process further, an area that is deemed to be optimally suited to a given form of eco-industrial development could be directly compared to areas categorized as high, medium, and, where appropriate, low suitability to determine what is 
'missing' from these areas that would allow them to achieve industrial symbiosis 'conducive environment' status. Similarly, by generating variables that constitute known fundamental (symbiosis) niches for particular industry types, an SSM could be used to strategically plan the industrial development of an area, both in relation to existing industry and inward investment enquiries. Instead of rhetoric based aspirational development that is prevalent within many regions' spatial planning strategies, a niche based SSM would inform the enduser as to exactly what forms of industry an area is inherently suited to in relation to realizing opportunities for regional resource efficiency.

It could be beneficial to incorporate more variables into an SSM that are not necessarily industrial characteristics of the baseline. For example, in terms of characterizing social or technological enablers of industrial symbiosis, the existence and coverage of an existing symbiosis or other form of industry focused social network, proximity to a work force, or access to points of resource innovation (e.g., a major city, companies with research and development facilities, or a university) could also be included within an MCE suitability map. In a similar vein, it could be beneficial, if suitable data are available, to incorporate a 'habitat' trajectory aspect into the SSM by including variables relating to planned industrial developments. This would, among other objectives, allow some flexibility to be incorporated into the SSM in terms of allowing areas that are currently deemed to be of high suitability to be reclassified as optimum suitability if the area is undergoing development that will change its character to closer to that of the baseline.

Is it possible to transfer the presented methodology for the utilities focused SSM and the nascent materials focused SSM out of England? With context specific development of the SSM methodology to make it applicable to other countries via the identification and characterization of a suitable baseline 'habitat', the cost and time efficient deployment of experienced industrial symbiosis practitioners directly to areas of high synergy facilitation 
potential would be possible. Suitable geographic data permitting, it is our contention that this could fast track the implementation of industrial symbiosis and the development of ecoindustrial networks similar to those facilitated by NISP and those witnessed in places such as Kalundborg.

\section{Summary and Conclusions}

Possessing an understanding of the wider environment in which a given organism (or company) exists and proliferates provides a deeper insight into the past and potential evolution of the given individual. Context specific ecological research, it has been argued, is paramount to delivering tangible industrial ecology (Jensen et al. 2011b). An SSI assessment, which is visualized via multi-criteria-evaluation mapping in the form of an SSM, attempts to present the end-user with a wider understanding of the propensity of a given industrial system to evolve toward greater resource efficiency. Instead of initially concentrating on the individual and their needs, the SSI process encourages the assessment of industrial ecosystems as a whole, and thus points end-users toward areas to initiate industrial symbiosis facilitation efforts. Once both the restrictions and opportunities for system evolution are fully understood within a given area, it is possible to determine which individuals are able to realize any uncovered niches, and the process of individual business engagement can begin. In effect, this promotes the organic evolution of context appropriate industrial symbiosis rather than the contrived industrial symbiosis that is promoted within 'greenfield' ecoindustrial parks that, based on existing evidence, tend to fail.

This article has demonstrated the process of producing a utilities sharing SSI that is aimed at comparing areas similarly prime for industrial symbiosis facilitation as the SHB. The SSM, derived from a geospatial characterization of the SHB, has pointed NISP practitioners toward further areas within England that outwardly possess the same geographic 
industrial attributes as the baseline 'habitat'. Although further research is clearly required, initial investigation of these areas and discussion with local stakeholders has tentatively shown them to be high in opportunities for eco-industrial development akin to those that exist within the baseline. The ability of the SSM to direct planners toward industrial symbiosis 'conducive environments' supports its proposed value as a resource efficiency decisionsupport tool within England. There are many options for the continued development and application of SSIs and SSMs to aid context sensitive industrial symbiosis planning. The existence and availability of datasets that provide sufficient information on a chosen SSM variable is, and will always remain, a constraint on the production of GIS based tools; however, it is hoped that the SSM methodologies presented within this article can be developed and transferred to other countries to support suitable data collection and the proactive identification of opportunities for industrial symbiosis internationally.

\section{Acknowledgements}

The presented article forms one element of doctoral research being conducted into regional resource planning by Paul D. Jensen. The Regional Resource Planning project is financially supported by the United Kingdom's Engineering and Physical Sciences Research Council and International Synergies Ltd. The authors would like to thank the three anonymous reviewers for their insightful comments and helpful suggestions for improving the article.

\section{References}

Chertow, M.R. 2007. "Uncovering” industrial symbiosis. Journal of Industrial Ecology 11(1): 11-30. 
DECC. 2008. Emissions Trading Scheme: National Allocation Plan 2008-12, HM Government Department of Energy and Climate Change. Available from: http://www.decc.gov.uk/en/content/cms/emissions/eu_ets/euets_phase_ii/phaseii_nap/ phaseii_nap.aspx

Ehrenfeld, J. and N. Gertler. 1997. Industrial ecology in practice: the evolution of interdependence at Kalundborg, Journal of Industrial Ecology 1(1): 67-80.

EA (Environment Agency). 2005. Integrated Pollution, Prevention and Control (Controlled Water) (C) Environment Agency database right 2008. Available from: EA National Customer Contact Centre, PO Box 544, Rotherham, UK, S60 1BY. http://www.environment-agency.gov.uk/contactus/

EA (Environment Agency). 2008a. Integrated Pollution, Prevention and Control (NonRecovered Industrial Wastes) (C) Environment Agency database right 2009. Available from: EA National Customer Contact Centre, PO Box 544, Rotherham, UK, S60 1BY. http://www.environment-agency.gov.uk/contactus/

EA (Environment Agency). 2008b. Live Borehole Licenses (C) Environment Agency database right 2008. Available from: EA National Customer Contact Centre, PO Box 544, Rotherham, UK, S60 1BY. http://www.environment-agency.gov.uk/contactus/

Gibbs, D. 2003. Trust and networking in interfirm relations: the case of eco-industrial development. Local Economy 18(3): 222-236. 
Gibbs, D. and P. Deutz. 2005. Implementing industrial ecology? Planning for eco-industrial parks in the USA. Geoforum 36(4): 452-464.

Gibbs, D. and P. Deutz. 2007. Reflections on implementing industrial ecology through ecoindustrial park development. Journal of Cleaner Production 15(17): 1683-1695.

Jensen, P.D., L. Basson, E.E. Hellawell, M.R. Bailey and M. Leach. 2011a. Quantifying 'geographic proximity': experiences from the United Kingdom's National Industrial Symbiosis Programme. Resources, Conservation and Recycling 55(7): 703-712.

Jensen, P.D., L. Basson and M. Leach. 2011b. Reinterpreting industrial ecology. Journal of Industrial Ecology 15(5): 680-692.

Kincaid, J. and M. Overcash. 2001. Industrial ecosystem development at the metropolitan level. Journal of Industrial Ecology 5(1): 117-126.

Kliskey, A.D., E.C. Lofroth, W.A. Thompson, S. Brown and H. Schreier. 1999. Simulating and evaluating alternative resource-use strategies using GIS-based habitat suitability indices. Landscape and Urban Planning 45(4): 163-175.

Laybourn, P. and M. Morrissey. 2009. National Industrial Symbiosis Programme: the pathway to a low carbon economy. Birmingham: International Synergies Ltd. 
Massard G. and S. Erkman. 2007. A regional industrial symbiosis methodology and its implementation in Geneva, Switzerland. Paper presented at the 3rd International Conference on Life Cycle Management, 27-29 August, Zurich.

Massard, G., A. Viquerat and S. Erkman. 2009. Detecting industrial symbiosis using a GIS based tool. Presentation to the $5^{\text {th }}$ International Conference on Industrial Ecology, 2124 June, Lisbon.

Mirata, M. 2004. Experiences from early stages of a national industrial symbiosis programme in the UK: determinants and coordination challenges. Journal of Cleaner Production 12(8-10): 967-983.

Nobel, C.E. and D.T. Allen. 2000. Using geographic information systems (GIS) in industrial water reuse modelling. Process Safety and Environmental Protection 78(B4): 295303.

Ordnance Survey. 2009. United Kingdom 'Strategi' (1:250,000), GIS dataset. (C) Crown Copyright/database right 2009. An Ordnance Survey/EDINA supplied service.

Özyurt, D.B. and M.J. Realff. 2002. Combining a geographical information system and process engineering to design an agricultural-industrial ecosystem. Journal of Industrial Ecology 5(3): 13-31.

USFWS (U.S. Fish and Wildlife Service). 1981. Standards for the development of habitat suitability index models. ESM 103. Washington, DC: Division of Ecological Services. 
Wakeley, J.S. 1988. A method to create simplified versions of existing habitat suitability index (HSI) models. Environmental Management 12(1): 79-83.

\begin{abstract}
About the Authors
Paul D. Jensen is a research engineer at NISP and the University of Surrey's Centre for Environmental Strategy (CES) in Guildford, Surrey, UK. Lauren Basson is a lecturer at CES. At the time the article was written, Emma E. Hellawell was a lecturer within the University of Surrey’s School of Civil Engineering. Matthew Leach is a professor and director of CES.
\end{abstract}

\footnotetext{
Notes

${ }^{1}$ One metric tonne $\approx 0.984$ imperial long ton or 1.102 imperial short ton.

${ }^{2}$ It is not the intention of this article to provide an in-depth description of the development and scope of traditional HSIs. For a detailed discussion on their origin, development, and wider application, please refer to USFWS (1981) and the numerous articles that exist on the subject within applied ecology journals.

${ }^{3}$ One square kilometer $\left(\mathrm{km}^{2}, \mathrm{SI}\right)=100$ hectares $($ ha $) \approx 0.386$ square miles $\approx 247$ acres.

${ }^{4}$ See the article by Mirata (2004) for a discussion on the development of an industrial symbiosis network within the wider Humber region.

5 The continual colonization of the site has also led to a reduction in the capacity of the SHB's power distribution network to transmit excess power production from companies that possess embedded generation.

${ }^{6}$ Fundamental niche: the entire range of niches a given organism (or company) can potentially fill in the absence of competition for resources.

${ }^{7}$ If there are more suitable areas in the UK for a baseline, this would not be of significant consequence for the purpose for which the SSI is being developed, since the baseline 'habitat' would identify these as being at least as suitable as the SHB.

${ }^{8}$ One metric meter (or metre) $\approx 1.09$ imperial yards or 3.28 imperial feet.
} 


\footnotetext{
${ }^{9}$ Raster format map layers are necessary for MCE overlay modeling because it is their ability to carry individual cell values that, once each map layer is aggregated, enables the relative SSI figure of a given area to be represented.

${ }^{10}$ Indeed, in comparison to the figures given in table 2, a sensitivity analysis performed on the use of different variable characterization distances showed that using the full distances given in table 1 resulted in increases in areas of total optimum, high, and medium suitability by $641.7 \%, 288.5 \%$, and $115.7 \%$, respectively; while the area allocated to the unsuitable habitat category decreased by $0.4 \%$.
} 